\title{
Emotional Exhaustion and Feeling Fed Up as the Driving Forces of Parental Burnout and its Consequences on Children: Insights from A Network Approach
}

This is a preprint (version May 24, 2022) of a manuscript that is currently being peerreviewed for publication in a scientific journal. Anonymized data, $\mathrm{R}$ code, and all materials are available via the Open Science Framework: https://osf.io/a89sd/.

Rana Begum Kalkan*1,2, M. Annelise Blanchard*2,3, Moïra Mikolajczak² ${ }^{2}$ Isabelle Roskam ${ }^{2}$, \& Alexandre Heeren ${ }^{2,3,4}$

${ }^{1}$ Katholieke Universiteit Leuven, Leuven, Belgium

${ }^{2}$ Psychological Sciences Research Institute, Université catholique de Louvain, Louvain-laNeuve, Belgium

${ }^{3}$ Belgian National Science Foundation (F.R.S.-FNRS), Brussels, Belgium

${ }^{4}$ Institute of Neuroscience, Université catholique de Louvain, Brussels, Belgium

\begin{abstract}
Author Note
* Contributed equally (i.e. shared first-authorship).

Rana Begum Kalkan (D) https://orcid.org/0000-0002-3321-916X

M. Annelise Blanchard (iD https://orcid.org/0000-0002-9605-7022
\end{abstract}


Moïra Mikolajczak (D) https://orcid.org/0000-0002-7333-1578

Isabelle Roskam (iD https://orcid.org/0000-0002-1449-1133

Alexandre Heeren (iD https://orcid.org/0000-0003-0553-6149

The data analysis for this study was preregistered on the Open Science Framework (https://osf.io/vqmzb). The anonymized summed data, as well as the R code used for analyses, are also available on the Open Science Framework (https://osf.io/a89sd/).

Moïra Mikolajczak. and Isabelle Roskam have founded the "Training Institute for Parental Burnout", which delivers training on parental burnout to professionals. The institute did not participate in the funding of this study, nor did it influence the process or the results in any manner. Alexandre Heeren receives honoraria for his editorial work from Elsevier. The other authors have no known conflict of interest to disclose.

M. Annelise Blanchard, Moïra Mikolajczak, Isabelle Roskam, and Alexandre Heeren were supported by a Coordinated Research Grant ("BParent") from the French Community of Belgium (ARC Grant 19/24-100). Alexandre Heeren (FRS-FNRS Research Associate, Grant 1.C.059.18F) and M. Annelise Blanchard (Research Fellow) are both also funded by the FRSFNRS Belgian National Science Foundation. These funds did not exert any influence or censorship of any kind on the present work.

Correspondence concerning this article should be addressed to M. Annelise Blanchard. Psychological Sciences Research Institute, Université Catholique de Louvain, Place du Cardinal Mercier, 10, B-1348, Louvain-la-Neuve, Belgium. E-mail: marie.blanchard@uclouvain.be 


\begin{abstract}
Parental burnout results from chronic stress in parenting, and it can be accompanied by harmful behaviors such as parental neglect and violence (Mikolajczak \& Roskam, 2018). Network analysis examines psychological phenomena within a system of its constituents, and thus it is promising for understanding the distinct features of parental burnout and behaviors related to it. Recently, Blanchard et al. (2021) conducted the first network analysis of parental burnout and related harmful behaviors in the family context, but did so using an outdated measure and conceptualization of parental burnout. In the present study, in a sample of Frenchspeaking parents ( $\mathrm{N}=3144$, from five different previous studies), we aimed to investigate how each of the four features in the new conceptualization of parental burnout (i.e., emotional exhaustion, feeling fed up, emotional distance, and contrast with the previous parental self) interact with one another and with parental neglect and violence in a network system. In this preregistered reanalysis, we generated two network models commonly used with cross-sectional data: a Graphical Gaussian Model and a Directed Acyclic Graph. Our results point to emotional exhaustion and feeling fed up as key driving forces of the network structure, while emotional distance appears as a critical feature tying parental burnout with parental neglect and violence.
\end{abstract} Keywords: parental burnout, network analysis, neglect, violence, directed acyclic graph 


\section{Emotional Exhaustion and Feeling Fed Up as the Driving Forces of Parental Burnout and its Consequences on Children: Insights from A Network Approach}

Parental burnout is a clinically significant condition arising from a long-term imbalance between parental stressors and parental resources (Mikolajczak \& Roskam, 2018; Roskam et al., 2021). Parents experiencing parental burnout feel emotionally exhausted, distant from their children, fed up with being a parent, and a sense of contrast with the parent they were beforehand. Its prevalence estimates are considerably high worldwide, rising to 7 to $8 \%$ in countries with the highest rates (e.g., Belgium, Poland, the USA; Roskam et al., 2021)

Investigating parental burnout is especially important as it can be damaging for all individuals in the family system. Most crucially, parental burnout strongly increases parental neglect and violence towards children. This effect appears causal, supported by cross-lagged results from two independent samples (both reported in Mikolajczak et al., 2019) and a recent experimental intervention study (Brianda et al., 2020a). When parental burnout is treated, child neglect and violence decrease proportionally to the decrease in parental burnout symptoms. The effect of parental burnout on parental behaviors requires special attention, as neglect and violence have widespread, long-term outcomes on children's development (Delima \& Vimpani, 2021; National Scientific Council on the Developing Child, 2010). However, most of these previous parental burnout studies relied on a unitary approach by tallying the different constitutive features of parental burnout into one sum-score, thus ignoring any possibility that these distinct features could interact differently with child neglect and violence.

Recently, however, an approach focusing specifically on the interactions between variables has arisen: a network approach to psychopathology that conceptualizes mental disorders as network systems of interacting symptoms (Borsboom, 2017). From this perspective, 
instead of investigating mental disorders as reflecting a single, unitary construct, one can examine the structure of, and associations between, the symptoms themselves. Studying a mental disorder's network structure can thus grant new insight into its symptom-to-symptom associations and topology. Though only recently pioneered by Borsboom and colleagues (Borsboom, 2017; Borsboom et al., 2011; Borsboom \& Cramer, 2013), this approach has quickly grown prevalent in contemporary clinical psychological sciences. Many studies are now using this framework to investigate the interrelations between systems of symptoms and to speculate as to the clinical implications (for a general overview, see Blanchard \& Heeren, 2022; for systematic reviews, see Contreras et al., 2019; Robinaugh et al., 2020)

Moreover, using a network approach to model psychopathology allows the adoption of network tools and graph theory concepts to understand mental disorders. One of these network concepts is node centrality, a tenet which posits that some nodes (here, symptoms) in the network are more important to the network structure than others (e.g., Blanchard \& Heeren, 2022; McNally, 2016). Nodes are viewed as more central if they are especially connected to the rest of the network (i.e., sharing many strong connections with other nodes), because they can then influence the entire network. In network models, highly central nodes are thought to maintain the network structure since, once activated, they can quickly spread that activation to the entire system (e.g., Barabási \& Albert, 1999; Borgatti, 2005). Within the network approach to psychopathology, central nodes have accordingly been theoretically (Borsboom \& Cramer, 2013) and empirically (e.g., Elliott et al., 2019; Papini et al., 2019) linked to the prognosis of a disorder, although statisticians also advise caution when interpreting what exactly central nodes signify (Bringmann et al., 2019). 
Analyzing network structures can also give insight into whether nodes form into communities (i.e., clusters of nodes that are more connected to each other than other nodes) and whether any specific nodes act as bridges to connect these communities. According to the network theory, these 'bridge' nodes could, for example, trigger a transition from a cluster of specific psychopathology features to related harmful behaviors (Jones et al., 2019). Identifying communities and the nodes that connect them thus helps researchers understand how specific symptoms influence the network system.

A network approach is especially suitable for investigating parental burnout and its relationship to parental neglect and violence (Blanchard \& Heeren, 2020). A network approach views a psychological phenomenon as a dynamic system of interacting components (Borsboom \& Cramer, 2013), and parental burnout does not exist in isolation but instead arises through the interactions of many variables relating to the entire family. Indeed, parental burnout has been conceptualized as an imbalance of parental stressors (e.g., conflict with the spouse, family disorganization, inconsistent childrearing practices) as compared to parental resources (e.g., support from the spouse, consistent childrearing practices; Mikolajczak \& Roskam, 2018), highlighting that parental burnout is embedded within the family system. A network approach thus enables us to investigate the features of parental burnout and their associations with maladaptive behaviors in the family by visualizing these variables as a system of interacting nodes.

Recently, in a large cross-sectional network study $(n=1551)$, Blanchard et al. (2021) conducted the first network analysis to examine how the hallmark features of parental burnout interact with one another and with parental behaviors related to the partner and the child(ren). They used two distinct computational network approaches: a Graphical Gaussian Model (GGM), 
which is an undirected network in which nodes represent variables and edges signify the partial correlations between each pair of nodes (Epskamp \& Fried, 2018), and a Bayesian network method to estimate a directed acyclic graph (DAG), which encodes the conditional independence relationships between the variables of interest and wherein each edge has an arrow tip on one end, indicating the direction of probabilistic dependence between them (Heeren et al., 2020; McNally, 2016; McNally et al., 2017; Scutari, 2010). In the GGM, emotional distance was highly central, and strongly connected with the other parental burnout features and with neglectful and violent behaviors toward children. Moreover, a community detection analysis (implemented via the Spinglass algorithm) identified emotional distance forming a subcommunity with parental neglect and violence. These observations all proposed emotional distance as a key variable that may maintain parental burnout within the family system, and that specifically relates to parental neglect and violence. And these observations were echoed in the DAG, wherein emotional distance emerged as a probabilistic driving force of the entire network structure.

Yet, although Blanchard et al. (2021) offered insight into the interactions of parental burnout features and related harmful behaviors, extensive research has recently suggested a new conceptualization of parental burnout, which was not considered in Blanchard et al. (2021), nor in other recent network approaches to parental burnout that did not focus on behaviors toward children (i.e., Le Vigouroux et al., 2022). The original conceptualization of parental burnout arose from the job burnout literature, and focused on exhaustion, distance, and inefficacy as a parent. However, a new theoretical approach has focused on building a conceptualization of parental burnout directly from the qualitative experiences of burned-out parents (Hubert \& Aujoulat, 2018). This new framework pivoted from inefficacy as a parent (relating to the output 
of work) toward feeling fed up (relating more directly to parents' emotional experiences).

Crucially, this new framework also adds a new feature to parental burnout: parents report feeling a sense of contrast with their previous parental selves; they previously felt happy and invested in parenting, and now experience shame and guilt that they have lost that investment in their parenting role (Roskam et al., 2018). Many studies have provided support for this new framework of parental burnout (Aunola et al., 2020; Furutani et al., 2020; Matias et al., 2020; Sodi et al., 2020). In line with this new conceptualization, a new measurement (i.e., the Parental Burnout Assessment, PBA) has been developed and validated in many languages and cultures (e.g., Arikan et al., 2020; Gannagé et al., 2020; Szczygieł et al., 2020). Thus, understanding this new conceptualization and its relationship with parental neglect and violence through the lens of a network approach might set the scene for novel conceptual and translational avenues.

Considering that this new conceptualization has significant conceptual and translational value, we aimed to examine in an exploratory and data-driven way how these four features of parental burnout (i.e., exhaustion, emotional distance, feeling fed up, and contrast with the previous parental self) relate to neglectful and violent behavior towards the child(ren). With the new conceptualization of parental burnout, which pulls from parents' qualitative experiences with burnout and which has been validated in many cultures, does emotional distance still play a central role in maintaining the network, as it did in Blanchard et al. (2021)? Does emotional distance still connect strongly with parental neglect and violence? How do contrast with the previous parental self (an additional feature) and feeling fed up (an updated feature focusing on parents' emotional experience vs. their concrete parenting ability), both of which refer more to affective aspects of parental burnout, interact with the other parental burnout features? Crucially, how do all of these features interact with parental neglect and violence? We use two different 
network methods to answer all of these questions, so as to build a better understanding of this new conceptualization of parental burnout and how it relates to child maltreatment.

\section{Method}

This study was an exploratory reanalysis of secondary data. We preregistered the analysis plan, methodology, and prior knowledge of the dataset following the guidelines of Weston et al. (2019) at https://osf.io/vqmzb. Anonymized data and the R code can be accessed at https://osf.io/a89sd/. There were no deviations from the preregistration.

\section{Participants}

The data came from five different previous studies (Brianda et al., 2020) wherein four features of parental burnout (i.e., exhaustion, feeling fed up, emotional distance, and contrast with previous parental self) were assessed along with neglect and violence towards children. One of these studies is further detailed in Brianda et al. (2020), another in Bayot et al. (under review), and the remaining three are unpublished master's theses. The total dataset included 3144 Frenchspeaking parents (86.7\% women) who had at least one child living with them. The participants were recruited through internet advertisements, social media, online forums, and word of mouth. Further information about the data collection of the unpublished master's theses can be found in the Supplementary Materials. Readers can refer to Brianda et al. (2020) and Bayot et al. (under review) for detailed data collection procedures for those specific studies.

\section{Measurements}

\section{Parental Burnout}

The four features of parental burnout were assessed using the Parental Burnout Assessment (PBA; Roskam et al., 2018). The PBA has four subscales, each referring to one of the four features of parental burnout: emotional exhaustion (9 items; e.g. I find it exhausting just 
thinking of everything I have to do for my children), emotional distance towards child(ren) (3 items; e.g. I do what I'm supposed to do for my child(ren), but nothing more), feelings of being fed up (5 items; e.g. I don't enjoy being with my children), and contrast with the previous parental self (6 items; e.g. I tell myself that I'm no longer the parent I used to be). Participants rated items on a 7-point Likert frequency scale, from never (0) to always (6). In this dataset, reliability estimates of the global score and each subscale were good: the Cronbach's alpha for the global score was .97 (.95 for emotional exhaustion, .81 for emotional distance, .93 for feelings of being fed up, and .93 for contrast with the previous parental self). For our analyses, we calculated the mean ${ }^{1}$ of each subscale separately as we were interested in the four features of parental burnout. This meant that the scores for each subscale ranged from 0 to 6 , with higher scores indicating higher levels of parental burnout.

\section{Parental Neglect}

Parental neglect was measured with the Parental Neglect Scale (17 items) (Mikolajczak et al., 2018), which measures physical, educational, and emotional neglect. All items assessing parental neglect were rated on an 8-point frequency scale, from never (0) to several times a day (7). Two of the studies in this dataset (studies \#2 and \#4) assessed parental neglect with a threeitem short form of this questionnaire which involved one representative item from each category of neglect. The other three studies (studies \#1, \#3, and \#5) assessed the full versions of the scale; we used only items corresponding to the short form from these studies (for more details, see the supplementary materials). The Cronbach's alpha for the neglect scale of three items was .62.

\section{Parental Violence}

\footnotetext{
${ }^{1}$ When administering the PBA, a global score is calculated by summing all items. However, we were interested in the subscale scores, and for the purposes of our network analyses we wanted all subscale scores to be on the scale, and so we calculated the mean of each subscale. In doing so, we followed our preregistration.
} 
Parental violence was measured with the Parental Violence Scale (15 items)

(Mikolajczak et al., 2018) encompassing verbal, physical, and psychological violence. Items were rated on an 8-point frequency scale, never (0) to several times a day (7). As in parental neglect, two studies (studies \#2 and \#4) used the three-item questionnaire of parental violence and three studies (studies \#1, \#3, and \#5) used the full version, although we only used the items corresponding to the short scale from these. The Cronbach's alpha value for the violence scale of three items was .57. For further information on how the short and long form questionnaires of parental neglect and violence were combined to form the nodes for "Neglect" and "Violence", see the supplementary materials.

\section{Network Analysis}

\section{Data Preparation}

Our analysis included six nodes, each representing a different variable: the four main features of parental burnout (i.e., emotional exhaustion, emotional distance, feeling fed-up, and contrast), as well as parental violence and parental neglect. We nonparanormally transformed all variables using the R package huge (Jiang et al., 2019). This ensured that the data met the assumption of multivariate normality required by the GGM, as well as ensured that each node had the same distribution, since different nodes were assessed with different scales. Because non-positive definite matrices can result in densely connected networks that may include false positives and unexpected negative edges (Epskamp \& Fried, 2018), we verified that our correlation matrix was positive definite, thereby confirming that nodes were not a linear combination of one another. Finally, we investigated whether any of the nodes were redundant or highly overlapping in content: we sought whether any nodes had high intercollinearity (i.e., $r>$ $.50)$ and similar correlations with other variables (i.e., only $25 \%$ of correlations significantly 
differing for a specific pair of nodes). To this end, we followed previous research (e.g., Bernstein et al., 2019) and implemented the Hittner method for comparing dependent correlations (Hittner et al., 2003) via the goldbricker function from the networktools package. We did not identify any redundant nodes.

\section{Graphical Gaussian Model}

We used the Graphical Gaussian Model (GGM) to investigate how the features of parental burnout and parental violence and neglect interact in a network system. We regularized the GGM via the LASSO (Least Absolute Shrinkage and Selection Operator) algorithm. The LASSO algorithm computes regularized partial correlations between each pair of nodes and thus limits the number of spurious edges due to associations with other nodes. It also shrinks small trivial associations (edges) to zero, thereby minimizing false positive edges and leaving a network with only strong edges (Friedman, Hastie, \& Tibshirani, 2014).

We used the qgraph package to implement LASSO regularization together with the Extended Bayesian Information Criterion (EBIC) model selection (Foygel \& Drton, 2011), which together have been shown to result in networks with high specificity (Epskamp \& Fried, 2018). This procedure generates 100 models with varying degrees of sparsity, and the model with the lowest EBIC value is selected as the final model, given a specific hyperparameter $(\gamma)$. This hyperparameter $\gamma$ determines the balance between including false-positive edges and excluding true edges. The hyperparameter $\gamma$ is typically set between 0 and 0.5 : a hyperparameter closer to 0.5 results in the EBIC favoring a simpler model with fewer edges, while setting the value for $\gamma$ closer to 0 leads the EBIC to favor models with more edges. To ensure that the edges in our network are true, we set this hyperparameter $(\gamma)$ to 0.5 , as recommended by simulation studies (Epskamp \& Fried, 2018). We estimated the stability of the edges using bootstrapped 
confidence intervals for each edge by generating 1,000 bootstrapped samples via the bootnet package (Epskamp \& Fried, 2018).

To assess the importance of each node to the network, we computed the expected influence centrality of each node. Expected influence is the absolute sum of negative and positive edge weights connected to a particular node. Higher expected influence values signify greater centrality and importance in the network. We used the case-dropping subset bootstrap framework (Epskamp et al., 2018) to evaluate the stability of centrality estimates. In this framework, the centrality estimates are assumed to be stable if they remain the same or highly similar even after dropping a certain amount of cases or nodes (Costenbader \& Valente, 2003). We measured the central stability coefficient (CS-coefficient) to quantify the centrality stability (Epskamp et al., 2018).

Lastly, we investigated whether the features of parental burnout and parental neglect and violence cohere as a network system, or whether they cluster within subnetworks (e.g., one community with parental burnout features and another community with parental neglect and violence). Following previous research, we implemented the spinglass algorithm (Reichardt \& Bornholdt, 2006), a community detection procedure suited for relatively small networks with positive and negative edge-weight values (e.g., Traag \& Bruggeman, 2009). We used the spinglass.community function of igraph to run the algorithm (Csardi \& Nepusz, 2006). We also investigated the presence of bridge nodes, or nodes that connect one community to another. We did so by computing the bridge expected influence of each node, that is, the absolute sum of positive and negative edge weights connecting a given node to all nodes in the other community or communities. These 'bridge' nodes are theorized to have clinical significance (Jones et al., 
2019), since they could, for example, trigger an activation from one cluster of nodes (e.g., of parental features) to another cluster of nodes (e.g., of parental neglect and violence).

\section{Directed Acyclic Graph}

Finally, we computed the DAG by implementing the Bayesian hill-climbing algorithm via the bnlearn package (Scutari, 2010; Scutari \& Denis, 2015). This algorithm computes different combinations of edges (i.e., adding, removing, and reversing their direction) and finds the best fitting model according to a goodness-of-fit target score (i.e., the Bayesian Information Criterion, or BIC). This iterative procedure is randomly restarted with different possible edges and node pairs, random restarts (to avoid local maxima), and system perturbations (i.e., adding, removing, or reversing an edge), and eventually yields the best fitting network according to this iterative process. We set the parameters to 50 random restarts and 100 perturbations, following previous research (Bernstein et al., 2019; McNally et al., 2017).

To check the stability of the DAG, we bootstrapped 10,000 samples (with replacement) and averaged across the networks resulting from these bootstrapped samples. In a first step, represented in one figure, we determined the frequency of a particular edge's presence in the bootstrapped networks. Then we set a criterion according to the optimal cut-point method of Scutari and Nagajaran (2013) for edge retention. In the second step, represented in a second figure, we ensured that the directions of each remaining edge were reliable in the 10,000 bootstrapped networks. Following the recommendations of Scutari and Nagajaran (2013), if an edge pointed from one node to another in $51 \%$ of the bootstrapped networks at minimum, we included that directed edge in the final network. Of note, very thin edges (closer to 50\%) were nearly equally likely to point in either direction, and so they might represent bidirectional processes (McNally et al., 2017). 


\section{Results}

Descriptive statistics (i.e., mean, standard deviation, skewness, and kurtosis) of the four features of parental burnout and parental neglect and violence (before the nonparanormal transformation) can be found in the supplementary materials. Pearson product-moment correlations between parental burnout and parental neglect and violence variables after the variables were nonparanormally transformed were comparable in size to the previous studies by Blanchard et al. (2021), varying between .37 and .47 depending on the parental burnout subscale considered (see Figure 1).

\section{Graphical Gaussian Model}

The resulting GGM network (regularized with graphical LASSO) is represented in Figure 2. Each node represents a variable, and the edges between nodes represent the regularized partial correlations between variables. There were no negative correlations, as all edges are colored in green. Several aspects of the network stand out. First, the largest edge weight connects exhaustion and feeling fed up, with an edge weight of $r=.62$. Second, all other edges are much smaller, with the second-largest edge weight, connecting contrast and emotional distance, having an edge weight of only $r=.27$. We conducted nonparametric bootstrapping and edge-weight difference tests to assess the stability and accuracy of each edge (see Figures S2 and S3 in the supplementary materials) and we concluded that the edge weights are stable: confidence intervals around each edge were small, and large edges significantly differed from smaller ones.

Next, we computed expected influence centrality. As can be seen in Figure 3A, feeling fed up had the highest expected influence, followed by exhaustion, and then by emotional distance and contrast with the previous parental self. 
To determine the subcommunities within the network, we used the Spinglass algorithm. The algorithm detected two subcommunities: a first community including the four parental burnout features (i.e., exhaustion, feeling fed up, emotional distance, and contrast with the previous parental self), and a second community encompassing parental neglect and parental violence toward the child(ren). Nodes with the highest bridge expected influence were parental violence and parental neglect as well as emotional distance (see Figure 3B). Both expected influence and bridge expected influence values were considerably stable, according to the casedropping bootstrapping method (see Supplementary Materials Figure 4).

\section{Directed Acyclic Graph}

The DAGs that result from the average of 10,000 bootstrapped networks can be seen in Figures 4A and 4B, each representing one step in generating the DAG (first, in Figure 4A, the presence of edges; second, in Figure 4B, their direction). Figure 4A thus reveals the importance of each edge to the network system, illustrating which edges are most likely to connect the different nodes. Thicker edges are more important for model fit (McNally, 2016), as edge thickness specifically represents the change in Bayesian Information Criterion (BIC, measuring a model's relative goodness-of-fit to the data) when that edge is taken out of the model. The edge connecting exhaustion and feeling fed up is the thickest, and thus the most important edge. The edges that connect exhaustion to contrast as well as feeling fed up to contrast also appear crucial for the network structure.

The edges in Figure 4B illustrate directional probabilities: a thicker edge represents an edge that was directed from node $\mathrm{X}$ to node $\mathrm{Y}$ in a greater proportion of the 10,000 bootstrapped networks. All of the edges in Figure 4B have similar low directional probabilities (see Supplementary materials Table 2). The two thickest edges are pointed from exhaustion to 
distance with a directional probability of .58 (i.e., in 5,800 of the 10,000 bootstrapped networks, the edge pointed in this direction) and from feeling fed up to violence.

As for the DAG's structure, exhaustion appears at the top of the network and directly influences the other features of parental burnout. Together with exhaustion, feelings of being fed up contributes to contrast with the previous parental self; and contrast directly influences emotional distance. Regarding parents' detrimental behaviors, violence is influenced by contrast, feeling fed up, and neglect. Interestingly, the R package bnlearn prompted a warning that there is likely an edge pointing from distance to parental neglect; however, it was not included in the graph as it would introduce a feedback loop.

\section{Complementary Analyses}

We wanted to see whether there were differences between mothers and fathers in terms of how parental burnout features interact with one another and with parental neglect and violence. To do so, we compared the GGM network structure of mothers $(\mathrm{N}=2725)$ and fathers $(\mathrm{N}=$ 416), using the Network Comparison Test (van Borkulo et al., 2017). We found no significant differences in the connectivity, structure, or edge weights of these two networks.

In addition, since commentators have argued that conclusions about node centrality might be distorted when variables have drastically different variances (i.e. differential variability) (e.g., Terluin et al., 2016), we computed the correlations between the standard deviation and the centrality estimates of each variable. Standard deviations were correlated with both expected influence, $r=.91, t(4)=4.54, p=0.01$, as well as with bridge expected influence, $r=-.89, t(4)$ $=-5.06, p=0.015$. 


\section{Discussion}

In this study, we aimed to investigate how the features of parental burnout in its new conceptualization interact with each other and with parental neglect and violence through the lens of network analysis. To this end, we used undirected and directed network models: a GGM and a DAG.

Considering the results of both network models, exhaustion and feeling fed up and their relationship appear crucial for propagating activation in the network structure since 1) in both the GGM and the DAG, the strongest link is between these two variables, 2) feeling fed up has the strongest expected influence estimate (i.e., is most strongly connected to other nodes in the network), followed by exhaustion. The exhaustion component refers to being overwhelmed and fatigued by parenting-related tasks (e.g., I find it exhausting just thinking of everything I have to do for my child(ren)), while feeling fed up can be conceptualized as a reflection of high levels of exhaustion on parents' general affective experiences when parenting (e.g., I can't take being a parent anymore). Thus, it theoretically makes sense that being exhausted is often accompanied by feeling fed up.

In line with their theoretical distinction, parental burnout features and parents' harmful behaviors formed two distinct communities. The nodes that potentially connect these two communities, as indicated by high bridge expected influence values, are emotional distance and parental neglect and violence; these nodes, therefore, are likely to shift activation from one community (i.e., parental burnout) to another (i.e., parental maltreatment). This finding also aligns with the fact that emotional distance is the only node that has direct connections to all other nodes in the GGM. 
Regarding the DAG representing the directional probabilities, the thickest edges pointed from exhaustion to distance and from feeling fed up to parental violence. However, the directional probabilities were all quite low (ranging between .59 to .51; see Table S2), thus likely indicating bidirectionality — that is, the edges could be pointing from either node to the other. For instance, the directional probability of the edge directed from contrast to distance was .52 , suggesting that emotional distance towards one's child(ren) can lead to a sense of contrast with the previous parental self as well as vice versa. Moreover, when computing the DAG, the R package bnlearn generated a message that an edge from emotional distance to parental neglect was also likely but not included in the graph, as it would introduce feedback loops to the DAG, which is constrained (as its name indicates) to be acyclic. This implies that parental neglect and emotional distancing might be reinforcing each other. These results together appear to be in line with family systems theory, which holds that an individual's cognitions and behaviors are embedded within a family context with all individuals influencing one another (Bavelas \& Segal, 1982). If a parent feels emotionally distant from their children, this can feed into a vicious circle that involves neglect or violence towards children. Indeed, between the likely bidirectionality of most edges in the DAG and the likely presence of feedback loops, it appears that for this specific dataset, a DAG is not optimal to model the relationship between variables. Instead, to be able to model and disentangle the bidirectional relationships between variables, other methods, such as measuring the variables over time and visualizing their connections in a temporal network, are necessary next steps.

Interestingly, these findings do not overall align with the results of the previous network analysis (Blanchard et al., 2021). In Blanchard et al. (2021), the driving force of the network was emotional distance in both the GGM and DAG models; here, however, feeling fed up and 
exhaustion seem to be the most important nodes for driving the system. This could be due to the differences in the conceptualization and measurement of parental burnout, as Blanchard et al. (2021) used the previous conceptualization of parental burnout, the Parental Burnout Inventory (Roskam et al., 2017). In the new conceptualization of parental burnout, the PBA (which was developed through an inductive qualitative approach from the experiences of parents), "feeling fed up" emphasizes the emotional component as opposed to the functional aspects of parental burnout (i.e., inefficacy in parenting) in the previous conceptualization. Contrast with the previous parental self is also a new addition focusing on the affective experiences of parents that is, feelings of guilt and shame that arise from parents' discrepancy between previous and current parental self. Our results, which highlight the key role of emotional exhaustion, support the Risks and Resources Theory of parental burnout which considers exhaustion as the starting point and a very important feature of parental burnout (Mikolajczak \& Roskam, 2018). According to this framework, a chronic imbalance between parenting risks (e.g. lack of partner support, poor coping strategies) and parenting resources gives rise to emotional and physical exhaustion which, in turn, initializes a cascade of negative thoughts and feelings towards one's self as a parent and one's child(ren). Both the results from this article and the theoretical framework for parental burnout therefore pinpoint emotional exhaustion as key to the development of parental burnout. Moreover, our results draw attention to the strong connection between exhaustion and lack of joy and fulfillment in parenting (i.e., feeling fed up) as central to the burnout experience. When viewed from a network perspective, which assumes that turning off a central node via targeted treatments can downgrade activation throughout the network (McNally, 2016), a critical next step should be to examine whether efforts to lessen parents' emotional exhaustion, perhaps via identifying and eliminating parenting risks and increasing 
internal and external resources, could lead to improvements in the entire parenting network system.

Although emotional exhaustion was central to the network system in the present article, a divergence from the only previous network study of parental burnout (Blanchard et al., 2021), there are also several aspects that overlap with this previous network study. Of note, emotional distance still seems to be a key feature that connects parental burnout with parental neglect and violence. Indeed, emotional distance had the highest bridge expected influence estimate among parental burnout features. Nodes with high bridge centrality are thought to shift activation from one community (here: parental burnout features) to another (parental maltreatment), increasing the likelihood of the latter occurring (Jones et al., 2019). Better understanding the role emotional distance plays within the parental burnout system, and potentially targeting it in interventions, therefore seems critical for preventing or eliminating parental neglect and violence.

Despite its strengths, this study had several limitations. First, our sample consisted of only French-speaking parents, which restricts the generalizability of the results, especially as there are different parenting styles typical to different cultures (Bornstein, 2012). For example, parents in Euro-American countries have intensifying parenting norms (Roskam et al., 2021), with representations of a "good parent" involving parents who are always warm and positive (with less focus placed on parenting discipline, firmness, or rules; Dupont et al., 2022). These high societal standards for parenting can pressure parents to reach for difficult or unattainable goals, which can lead to parental exhaustion (Kawamoto et al., 2018; Sorkkila \& Aunola, 2020). Other cultures that have different representations of a "good parent," or indeed different goals when raising a child, might exert less pressure on parents, and thereby less exhaustion (Bornstein, 2012). This could, for example, lead to a different relationship between parenting 
exhaustion and feeling fed up (currently the thickest edge in both networks). In addition, recent studies indicate that the rates of parental burnout vary significantly between cultures, particularly relating to individualism (Roskam et al., 2021). In addition, recent studies indicate that parenting and parental burnout vary significantly between cultures, particularly relating to individualism (Roskam et al., 2021). However, the effect-size of parental burnout on parental violence and neglect appears quite consistent across countries (see Mikolajczak et al., 2019 [Study 1, Study 2]; and Szczygieł et al., 2020) for correlations in Belgium, the UK, and Poland, respectively). Nonetheless, we encourage future studies to investigate the interactions between parental burnout features and related harmful behaviors in different cultures. Second, as in most research on parental burnout, our sample mainly included mothers $(86.7 \%)$. Although we did not find any differences between the network structure of mothers versus fathers, it would nonetheless be useful for future research to also investigate these questions with samples focusing on fathers. Third, the Cronbach's alphas of the short forms of the parental neglect and parental violence questionnaires were low (0.62 and 0.57 , respectively). Thus, future research should consider using other measures with higher reliability for parental neglect and violence, or at least the longer forms of these questionnaires. Fourth, we found that both the expected influence and bridge expected influence of nodes were correlated with the standard deviations of nodes. This implies that these estimates of node centrality could be distorted by the variables having differential variability (although the variables do not have drastically different standard deviations; see Table S1). Of note, since parental neglect and parental violence had high kurtosis values (see Table S1), these two variables might be driving this differential variability issue. On the other hand, it is not surprising that these variables are highly skewed in a large unselected sample. The interpretation of node centrality is generally under scrutiny with the psychological 
network literature (Bringmann et al., 2019) and as such the discussion of centrality for the GGM presented in this manuscript must therefore also be understood with caution.

\section{Conclusion}

The findings of this cross-sectional study pinpoint emotional exhaustion as the driving force of the network structure of parental burnout and parental neglect and violence, with feeling fed up also playing an influential role. This result aligns with the Risks and Resources theory of parental burnout (Mikolajczak \& Roskam, 2018), which considers exhaustion as the feature that first arises as the risks outweigh the resources of the parent. Emotional distance, on the other hand, emerges as a critical feature of parental burnout which is closely linked to parental neglect and violence, thus requiring special attention for preventing and intervening on these detrimental behaviors. As our results suggest, parental burnout and related harmful behaviors seem to occur in a vicious circle with potential bidirectional associations between many variables. Thus, future research on the temporal dynamics of these relationships is going to be a critical next step. 


\section{Table 1}

Data Collection: Data Sources, Sample Size, and Percentage of Female Participants

\begin{tabular}{llccc}
\hline Study & Source & N & \% Women & Mean Age (SD) \\
\hline 1 & Data collected for master's thesis (2021), not published & 1003 & 90.42 & $38.37(8.64)$ \\
2 & Data collected for master's thesis (2021), not published & 1538 & 84 & $42.99(8.31)$ \\
3 & Brianda et al. (2020) & 148 & 87.83 & $*$ \\
4 & Data collected for master's thesis (2021), not published & 381 & 86.87 & $38.90(7.48)$ \\
5 & Bayot et al. (under review) & 74 & 87.84 & $*$ \\
\hline
\end{tabular}

Note. ${ }^{*}=$ Not able to calculate mean ages or standard deviations; since these two studies were intervention studies with smaller samples, the authors decided to ensure the anonymity of participants by not collecting their exact age information, but instead age ranges. We therefore were unable to report the exact mean age and standard deviation for participants in these two studies, but interested readers can find the reported age ranges in the original manuscripts. 


\section{Figure 1}

Pearson Product-moment Correlation Plot for All Variables after Nonparanormal

Transformation

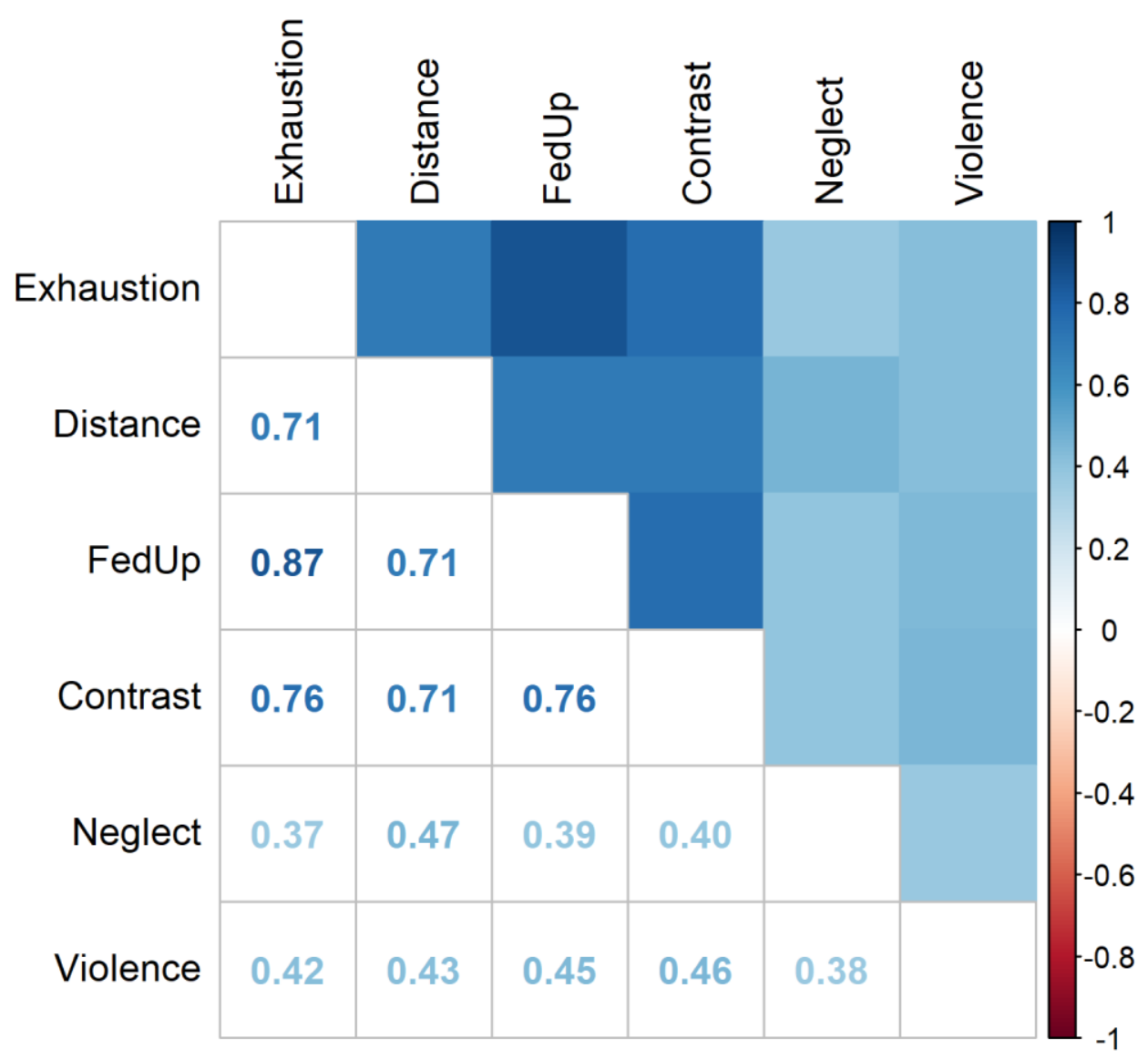

Note : Exhaustion = Emotional Exhaustion; FedUp = Feelings of being fed up Distance =

Emotional Distance; Contrast $=$ Contrast with the previous parental self; Neglect $=$ Parental neglect toward child(ren); Violence $=$ Parental violence toward child(ren) . 
Figure 2

\section{Graphical Gaussian Model structured with Graphical LASSO Algorithm}

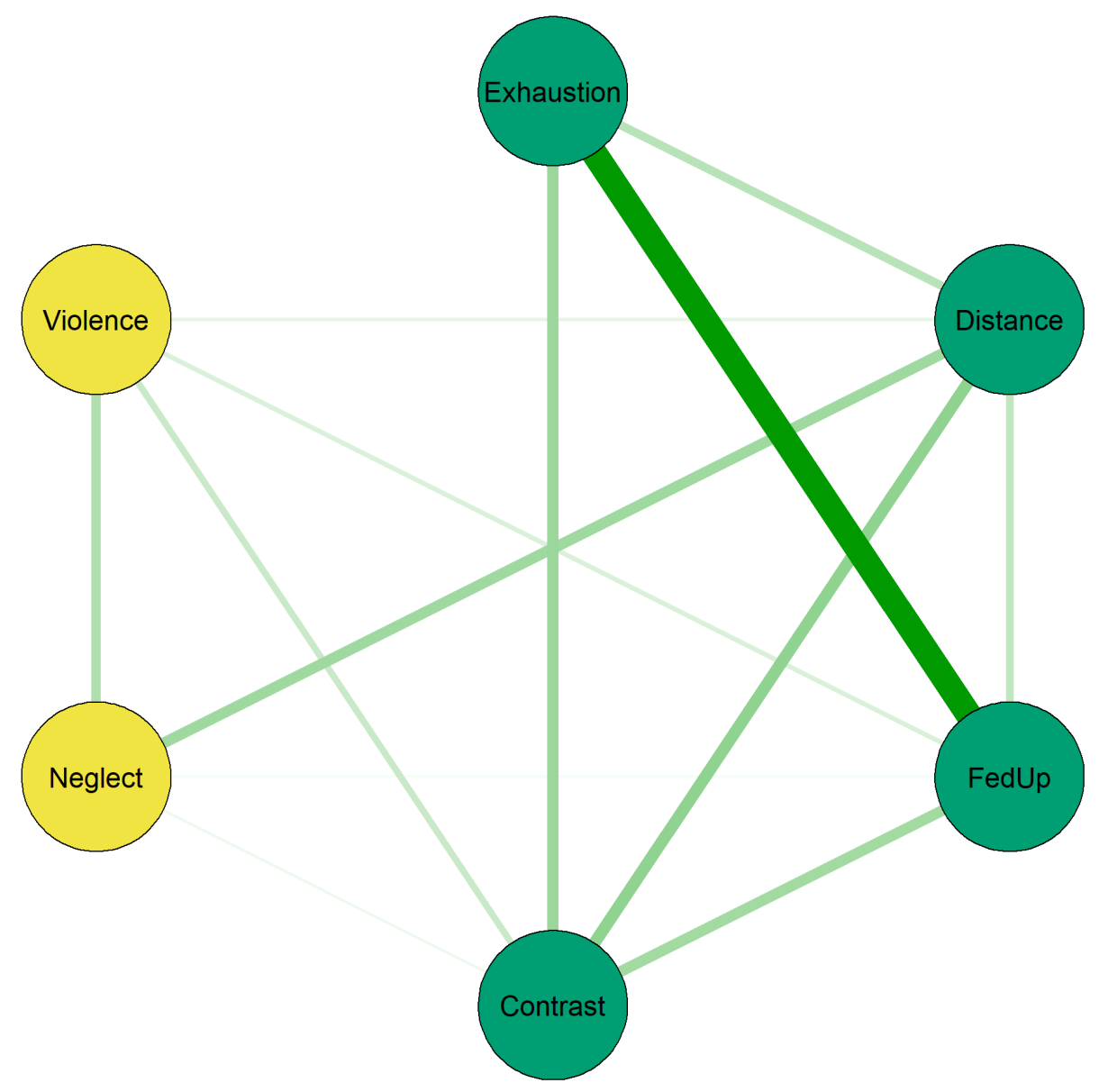

Note: The edge thickness represents the magnitude of regularized partial correlations, thicker edge implying higher partial correlation. Green edges denote positive regularized partial correlations. The different shades/colors represent different communities, as determined by the Spinglass algorithm. Exhaustion = Emotional Exhaustion; FedUp = Feelings of being fed up; Distance $=$ Emotional Distance Contrast $=$ Contrast with the previous parental self $;$ Neglect $=$ Parental neglect toward child(ren); Violence $=$ Parental violence toward child(ren) 


\section{Figure 3}

\section{Expected Influence and Bridge Expected Influence of Each Node}

A

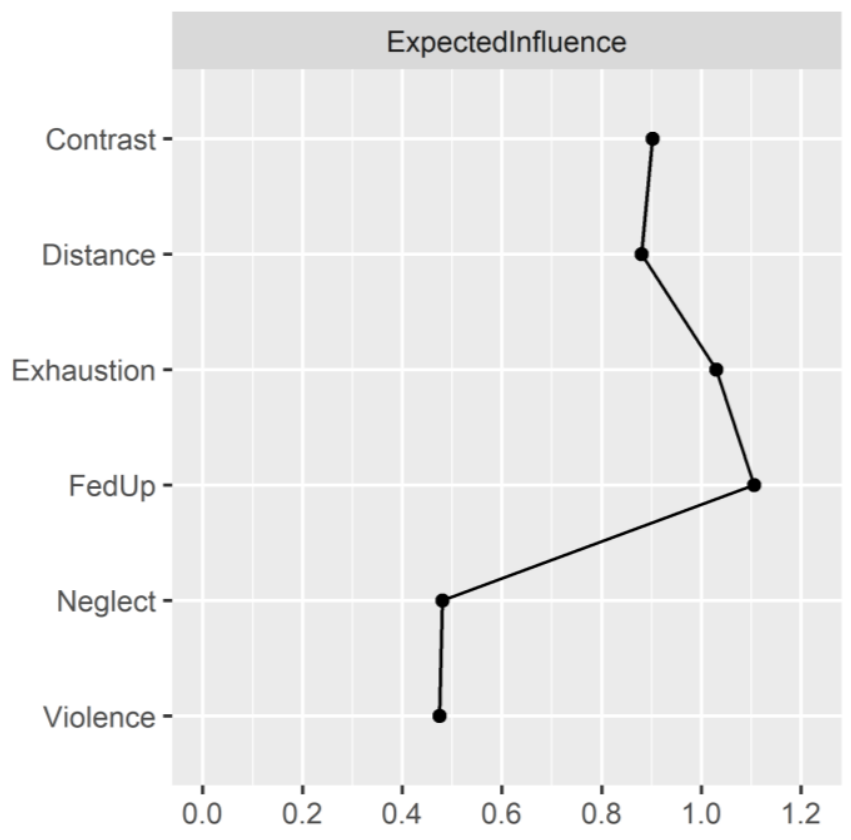

B

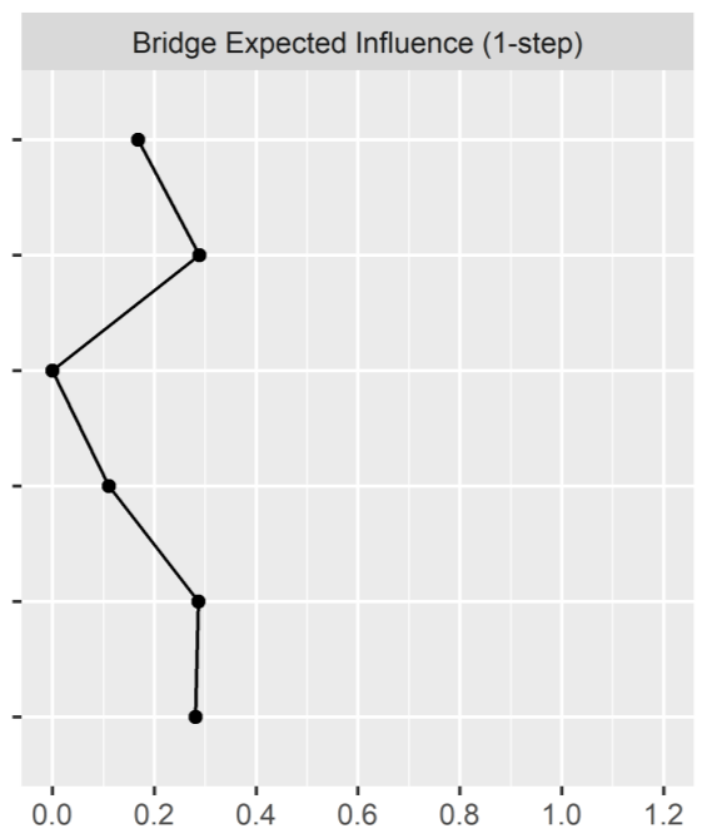

Note : Exhaustion = Emotional Exhaustion; FedUp = Feelings of being fed up; Distance = Emotional Distance; Contrast $=$ Contrast with the previous parental self , Neglect $=$ Parental neglect toward child(ren); Violence $=$ Parental violence toward child(ren) 


\section{Figure 4}

\section{Directed Acyclic Graphs}
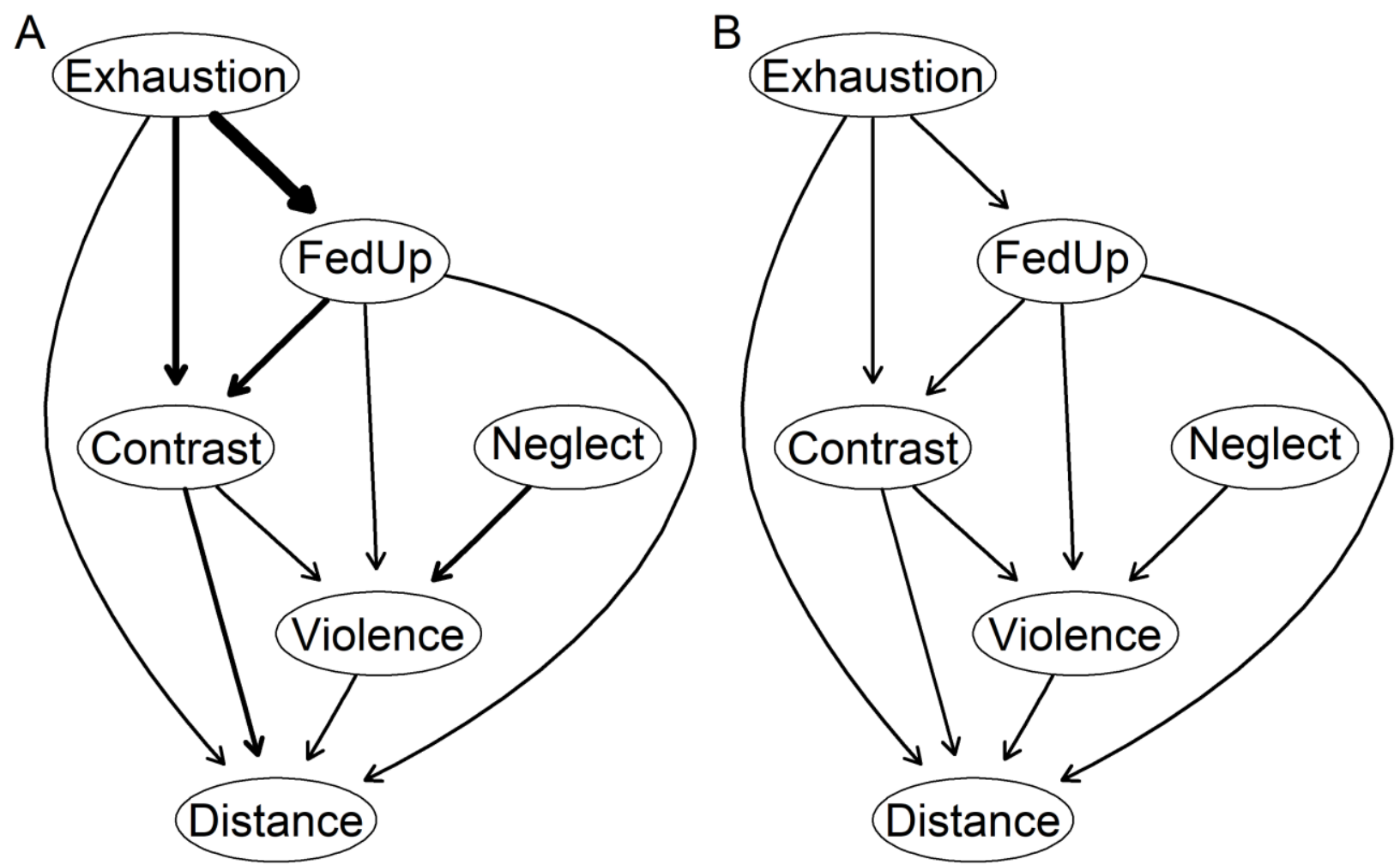

Note: Panel A (presence of edges): the thickness of the edges denotes the importance of the edge for the model fit, specifically, high edge thickness implies bigger change in the Bayesian Information Criterion (a model's relative goodness-of-fit for the data) when the edge is excluded from the model. Panel B (direction of edges): demonstrates directional probabilities of the edges, a thicker edge implying that it was directed in the represented fashion in a greater proportion of the 10,000 bootstrapped networks. Exhaustion = Emotional Exhaustion; FedUp = Feelings of being fed up; Distance $=$ Emotional Distance Contrast $=$ Contrast with the previous parental self; Neglect $=$ Parental neglect toward child(ren); Violence $=$ Parental violence toward child(ren) 


\section{References}

Arikan, G., Üstündăg-Budak, A. M., Akgün, E., Mikolajczak, M., \& Roskam, I. (2020). Validation of the Turkish version of the Parental Burnout Assessment (PBA). New Directions for Child and Adolescent Development, 2020(174), 15-32. https://doi.org/https://doi.org/10.1002/cad.20375

Aunola, K., Sorkkila, M., \& Tolvanen, A. (2020). Validity of the Finnish version of the Parental Burnout Assessment (PBA). Scandinavian Journal of Psychology, 61(5), 714-722. https://doi.org/https://doi.org/10.1111/sjop.12654

Barabási, A.-L., \& Albert, R. (1999). Emergence of Scaling in Random Networks. Science, 286(5439), 509 LP - 512. https://doi.org/10.1126/science.286.5439.509

Bavelas, J. B., \& Segal, L. (1982). Family Systems Theory: Background and Implications. Journal of Communication, 32(3), 99-107. https://doi.org/https://doi.org/10.1111/j.14602466.1982.tb02503.x

Bernstein, E. E., Heeren, A., \& McNally, R. J. (2019). Reexamining trait rumination as a system of repetitive negative thoughts: A network analysis. Journal of Behavior Therapy and Experimental Psychiatry, 63, 21-27. https://doi.org/10.1016/J.JBTEP.2018.12.005

Blanchard, A., \& Heeren, A. (2022). Ongoing and Future Challenges of the Network Approach to Psychopathology: From Theoretical Conjectures to Clinical Translations (pp. 32-46). https://doi.org/10.1016/B978-0-12-818697-8.00044-3

Blanchard, M. A., \& Heeren, A. (2020). Why we should move from reductionism and embrace a network approach to parental burnout. New Directions for Child and Adolescent Development, 2020(174), 159-168. https://doi.org/10.1002/cad.20377

Blanchard, M. A., Roskam, I., Mikolajczak, M., \& Heeren, A. (2021). A network approach to 
parental burnout. Child Abuse and Neglect, 111, 1-12. https://doi.org/10.1016/j.chiabu.2020.104826

Borgatti, S. (2005). Centrality and Network Flow. Social Networks, 27, 55-71. https://doi.org/10.1016/j.socnet.2004.11.008

Bornstein, M. H. (2012). Cultural Approaches to Parenting. Parenting, Science and Practice, 12(2-3), 212. https://doi.org/10.1080/15295192.2012.683359

Borsboom, D. (2017). A network theory of mental disorders. World Psychiatry, 16(1), 5-13. https://doi.org/10.1002/wps.20375

Borsboom, D., \& Cramer, A. O. J. (2013). Network Analysis: An Integrative Approach to the Structure of Psychopathology. Annual Review of Clinical Psychology, 9(1), 91-121. https://doi.org/10.1146/annurev-clinpsy-050212-185608

Borsboom, D., Cramer, A. O. J., Schmittmann, V. D., Epskamp, S., \& Waldorp, L. J. (2011). The Small World of Psychopathology. PLOS ONE, 6(11), 1-11. https://doi.org/10.1371/journal.pone.0027407

Brianda, M E, Roskam, I., Gross, J. J., Franssen, A., Kapala, F., Gérard, F., \& Mikolajczak, M. (2020). Treating Parental Burnout: Impact of Two Treatment Modalities on Burnout Symptoms, Emotions, Hair Cortisol, and Parental Neglect and Violence. Psychotherapy and Psychosomatics, 89(5), 330-332. https://doi.org/10.1159/000506354

Brianda, Maria Elena, Roskam, I., Gross, J. J., Franssen, A., Kapala, F., Gérard, F., \& Mikolajczak, M. (2020). Treating Parental Burnout: Impact of Two Treatment Modalities on Burnout Symptoms, Emotions, Hair Cortisol, and Parental Neglect and Violence. Psychotherapy and Psychosomatics, 89(5), 330-332. https://doi.org/10.1159/000506354 Bringmann, L. F., Elmer, T., Epskamp, S., Krause, R. W., Schoch, D., Wichers, M., Wigman, J. 
T. W., \& Snippe, E. (2019). What Do Centrality Measures Measure in Psychological Networks? Journal of Abnormal Psychology. https://doi.org/10.1037/ABN0000446

Contreras, A., Nieto, I., Valiente, C., Espinosa, R., \& Vazquez, C. (2019). The Study of Psychopathology from the Network Analysis Perspective: A Systematic Review. Psychotherapy and Psychosomatics, 88, 1-13. https://doi.org/10.1159/000497425

Costenbader, E., \& Valente, T. W. (2003). The stability of centrality measures when networks are sampled. Social Networks, 25(4), 283-307. https://doi.org/10.1016/S03788733(03)00012-1

Csardi, G., \& Nepusz, T. (2006). The igraph software package for complex network research. InterJournal Complex Systems, Complex Sy(1695), 1695. http://igraph.sf.net

Delima, J., \& Vimpani, G. (2021). The neurobiological effects of childhood maltreatment: An often overlooked narrative related to the long-term effects of early childhood trauma? Family Matters. https://doi.org/10.3316/ielapa.717010661945019

Dupont, S., Mikolajczak, M., \& Roskam, I. (2022). The Cult of the Child: A Critical Examination of Its Consequences on Parents, Teachers and Children. Social Sciences 2022, Vol. 11, Page 141, 11(3), 141. https://doi.org/10.3390/SOCSCI11030141

Elliott, H., Jones, P. J., \& Schmidt, U. (2019). Central Symptoms Predict Posttreatment Outcomes and Clinical Impairment in Anorexia Nervosa: A Network Analysis. Clinical Psychological Science, 8(1), 139-154. https://doi.org/10.1177/2167702619865958

Epskamp, S., Borsboom, D., \& Fried, E. I. (2018). Estimating psychological networks and their accuracy: A tutorial paper. Behavior Research Methods, 50(1), 195-212. https://doi.org/10.3758/s13428-017-0862-1

Epskamp, S., \& Fried, E. I. (2018). A tutorial on regularized partial correlation networks. 
Psychological Methods, 23(4), 617-634. https://doi.org/10.1037/met0000167

Foygel, R., \& Drton, M. (2011). Bayesian model choice and information criteria in sparse generalized linear models. https://doi.org/10.48550/arxiv.1112.5635

Friedman, J.H., Hastie, T., \& Tibshirani, R. (2014). Glasso: graphical lasso- estimation of Gaussian graphical models. $R$ package version 1.8 .

Furutani, K., Kawamoto, T., Alimardani, M., \& Nakashima, K. (2020). Exhausted parents in Japan: Preliminary validation of the Japanese version of the Parental Burnout Assessment. New Directions for Child and Adolescent Development, 2020(174), 33-49. https://doi.org/https://doi.org/10.1002/cad.20371

Gannagé, M., Besson, E., Harfouche, J., Roskam, I., \& Mikolajczak, M. (2020). Parental burnout in Lebanon: Validation psychometric properties of the Lebanese Arabic version of the Parental Burnout Assessment. New Directions for Child and Adolescent Development, 2020(174), 51-65. https://doi.org/10.1002/cad.20383

Heeren, A., Bernstein, E. E., \& McNally, R. J. (2020). Bridging maladaptive social self-beliefs and social anxiety: a network perspective. Journal of Anxiety Disorders, 74, 102267. https://doi.org/https://doi.org/10.1016/j.janxdis.2020.102267

Hittner, J. B., May, K., \& Silver, N. C. (2003). A monte carlo evaluation of tests for comparing dependent correlations. Journal of General Psychology, 130(2), 149-168. https://doi.org/10.1080/00221300309601282

Hubert, S., \& Aujoulat, I. (2018). Parental burnout: When exhausted mothers open up. Frontiers in Psychology, 9(JUN), 1-9. https://doi.org/10.3389/fpsyg.2018.01021

Jiang, H., Fei, X., Liu, H., Roeder, K., Lafferty, J., Wasserman, L., Li, X., \& Zhao, T. (2019). huge: High-dimensional undirected graph estimation (R package version 1.3.2) [Computer 
software].

Jones, P. J., Ma, R., \& McNally, R. J. (2019). Bridge Centrality: A Network Approach to Understanding Comorbidity. Multivariate Behavioral Research, O(0), 1-15. https://doi.org/10.1080/00273171.2019.1614898

Kawamoto, T., Furutani, K., \& Alimardani, M. (2018). Preliminary validation of Japanese version of the parental burnout inventory and its relationship with perfectionism. Frontiers in Psychology, 9(JUN), 970. https://doi.org/10.3389/FPSYG.2018.00970/BIBTEX

Le Vigouroux, S., Charbonnier, E., \& Scola, C. (2022). Profiles and age-related differences in the expression of the three parental burnout dimensions. European Journal of Developmental Psychology, 1-20. https://doi.org/10.1080/17405629.2021.1990749

Matias, M., Aguiar, J., César, F., Braz, A. C., Barham, E. J., Leme, V., Elias, L., Gaspar, M. F., Mikolajczak, M., Roskam, I., \& Fontaine, A. M. (2020). The Brazilian-Portuguese version of the Parental Burnout Assessment: Transcultural adaptation and initial validity evidence. New Directions for Child and Adolescent Development, 2020(174), 67-83. https://doi.org/https://doi.org/10.1002/cad.20374

McNally, R. J. (2016). Can network analysis transform psychopathology? Behaviour Research and Therapy, 86, 95-104. https://doi.org/10.1016/j.brat.2016.06.006

McNally, R. J., Heeren, A., \& Robinaugh, D. J. (2017). A Bayesian network analysis of posttraumatic stress disorder symptoms in adults reporting childhood sexual abuse. European Journal of Psychotraumatology, 8(sup3), 1341276. https://doi.org/10.1080/20008198.2017.1341276

Mikolajczak, M., Brianda, M. E., Avalosse, H., \& Roskam, I. (2018). Consequences of parental burnout: Its specific effect on child neglect and violence. Child Abuse and Neglect, 
80(March), 134-145. https://doi.org/10.1016/j.chiabu.2018.03.025

Mikolajczak, M., Gross, J. J., \& Roskam, I. (2019). Parental Burnout: What Is It, and Why Does It Matter? Clinical Psychological Science, 7(6), 1319-1329. https://doi.org/10.1177/2167702619858430

Mikolajczak, M., \& Roskam, I. (2018). A theoretical and clinical framework for parental burnout: The balance between risks and resources (BR2). Frontiers in Psychology, 9(JUN). https://doi.org/10.3389/fpsyg.2018.00886

National Scientific Council on the Developing Child. (2010). Early experiences can alter gene expression and affect long-term development: working paper. Center on the Developing Child at Harvard University, 1(10), 1-12. http://doi.wiley.com/10.1111/j.11512916.1918.tb17232.x

Papini, S., Rubin, M., Telch, M., Smits, J., \& Hien, D. (2019). Pretreatment PTSD symptom network metrics predict the strength of the association between node change and network change during treatment. Journal of Traumatic Stress. https://doi.org/10.1002/jts.22379

Reichardt, J., \& Bornholdt, S. (2006). Statistical mechanics of community detection. Physical Review E - Statistical, Nonlinear, and Soft Matter Physics, 74(1), 1-14. https://doi.org/10.1103/PhysRevE.74.016110

Robinaugh, D. J., Hoekstra, R. H. A., Toner, E. R., \& Borsboom, D. (2020). The network approach to psychopathology: a review of the literature 2008-2018 and an agenda for future research. Psychological Medicine, 50(3), 353-366. https://doi.org/10.1017/S0033291719003404

Roskam, I., Aguiar, J., Akgun, E., Arikan, G., Artavia, M., \& Avalosse, H. (2021). Parental Burnout Around the Globe : A 42-Country Study. Affective science, 2(1), 58-79. 
Roskam, I., Brianda, M. E., \& Mikolajczak, M. (2018). A step forward in the conceptualization and measurement of parental burnout: The Parental Burnout Assessment (PBA). Frontiers in Psychology, 9(JUN), 1-12. https://doi.org/10.3389/fpsyg.2018.00758

Roskam, I., Raes, M. E., \& Mikolajczak, M. (2017). Exhausted parents: Development and preliminary validation of the parental burnout inventory. Frontiers in Psychology, 8(FEB), 1-12. https://doi.org/10.3389/fpsyg.2017.00163

Scutari, M. (2010). Learning Bayesian Networks with the bnlearn R Package. Journal of Statistical Software; Vol 1, Issue 3 (2010) . https://www.jstatsoft.org/v035/i03

Scutari, M., \& Denis, J. (2015). Bayesian networks: With examples in R. Boca Raton, FL: CRC Press.Statistical Software; Vol 1, Issue 3 (2010) . https://www.jstatsoft.org/v035/i03

Scutari, M., \& Nagarajan, R. (2013). Identifying significant edges in graphical models of molecular networks. Artificial Intelligence in Medicine, 57(3), 207-217. https://doi.org/10.1016/J.ARTMED.2012.12.006

Sodi, T., Kpassagou, L. B., Hatta, O., Ndayizigiye, A., Ndayipfukamiye, J.-M., Tenkué, J. N., Bahati, C., \& Sezibera, V. (2020). Parenting and parental burnout in Africa. New Directions for Child and Adolescent Development, 2020(174), 101-117. https://doi.org/https://doi.org/10.1002/cad.20386

Sorkkila, M., \& Aunola, K. (2020). Risk Factors for Parental Burnout among Finnish Parents: The Role of Socially Prescribed Perfectionism. Journal of Child and Family Studies, 29(3), 648-659. https://doi.org/10.1007/S10826-019-01607-1/FIGURES/3

Szczygieł, D., Sekulowicz, M., Kwiatkowski, P., Roskam, I., \& Mikolajczak, M. (2020). Validation of the Polish version of the Parental Burnout Assessment (PBA). New Directions for Child and Adolescent Development, 2020(174), 137-158. 
https://doi.org/https://doi.org/10.1002/cad.20385

Terluin, B., De Boer, M. R., \& De Vet, H. C. W. (2016). Differences in Connection Strength between Mental Symptoms Might Be Explained by Differences in Variance: Reanalysis of Network Data Did Not Confirm Staging. PLOS ONE, 11(11), e0155205. https://doi.org/10.1371/JOURNAL.PONE.0155205

Traag, V. A., \& Bruggeman, J. (2009). Community detection in networks with positive and negative links. Physical Review E - Statistical, Nonlinear, and Soft Matter Physics, 80(3), 1-6. https://doi.org/10.1103/PhysRevE.80.036115

van Borkulo, C., van Bork, R., Boschloo, L., Kossakowski, J., Tio, P., Schoevers, R., Borsboom, D., \& Waldorp, L. (2017). Comparing network structures on three aspects: A permutation test. https://doi.org/10.13140/RG.2.2.29455.38569

Weston, S. J., Ritchie, S. J., Rohrer, J. M., \& Przybylski, A. K. (2019). Recommendations for Increasing the Transparency of Analysis of Preexisting Data Sets. Advances in Methods and Practices in Psychological Science, 2(3), 214-227.

https://doi.org/10.1177/2515245919848684 